BMJ Open Sport \& Exercise Medicine

\title{
Feasibility and effects of a home-based intervention using activity trackers on achievement of individual goals, quality of life and motor performance in patients with paediatric cancer
}

\author{
Miriam Götte, ${ }^{1,2}$ Sabine Verena Kesting, ${ }^{2,3,4}$ Joachim Gerss, ${ }^{5}$ Dieter Rosenbaum, ${ }^{6}$ \\ Joachim Boos ${ }^{2}$
}

To cite: Götte M, Kesting SV, Gerss J, et al. Feasibility and effects of a home-based intervention using activity trackers on achievement of individual goals, quality of life and motor performance in patients with paediatric cancer. BMJ Open Sport \& Exercise Medicine 2018;4:e000322. doi:10.1136/ bmjsem-2017-000322

- Additional material is published online only. To view please visit the journal online (http://dx.doi.org/10.1136/10. 1136/bmjsem-2017-000322).

MG and SVK contributed equally.

$M G$ and SVK are joint first authors.

Accepted 12 March 2018

\section{ABSTRACT}

Objectives Supervised exercise interventions during inpatient care are feasible. The objective was to evaluate the usability of activity trackers and centralised monitoring to conduct a home-based exercise intervention during cancer treatment. The primary endpoint and confirmatory analysis was achievement of individual goals for daily steps, compared (A) in the intervention group (IG) over time and (B) between the IG and control group (CG). Secondary endpoints included achievement of goals for active minutes and effects on motor performance and healthrelated quality of life (hrQoL)

Methods Forty patients treated for paediatric cancer (14.7 \pm 3.9 years) were included. The IG received a 6-8 week intervention during acute treatment (T1) and a 2-week intervention in transition to aftercare (T2). The CG only received the intervention at $\mathrm{T} 2$. Baseline tests to assess motor performance and physical activity were conducted prior to every intervention.

Results In the primary confirmatory analysis, the IG significantly improved achievement of individual step goals $(p=0.04)$ whereas group analyses did not reveal significant differences. Achievement of active minutes remained low $(p=0.23)$. IG scored higher in hrQoL than CG $(p<0.01)$ and percentage of children scoring below normative value in strength tests was higher in CG. Of all participants, $94 \%$ rated the intervention as meaningful and $80 \%$ as motivational.

Conclusions Results of this study indicate that this intervention for home stays with centralised supervision is feasible and leads to increased achievement of individual step goals. Despite the positive effects on hrQoL, further strategies are needed to increase positive effects on motor performance.

\section{INTRODUCTION}

Cancer diagnoses during childhood and adolescence are rare events; however, the life-threatening disease itself and the intensive multimodal therapy frequently lead to lifelong psychological and physical impairments. ${ }^{1-3}$ Motor function deficits are present
What are the new findings?

A home-based exercise intervention under centra supervision using activity trackers is feasible and accepted during and shortly after childhood cancer treatment.

Participants improve achievement of their individual step goals over time.

- The home-based intervention seems to increase health-related quality of life.

already at the beginning of cancer treatment ${ }^{4}$ and consist throughout the whole cancer trajectory. $^{5-7}$ Over the last years, physical activity interventions and specific exercise programmes have been identified as beneficial for adults with cancer due to their potential to reduce some negative side effects like fatigue ${ }^{8}$ or pain. ${ }^{9}$ Evidence for positive effects in patients with childhood cancer is still low, ${ }^{10-12}$ but feasibility of exercise programmes has been shown during in-hospital stays. ${ }^{1314}$ However, due to long distances between the families' homes and the specialised centres, supervised interventions are hardly feasible at home during the cycles between chemotherapy. Studies in adults with cancer using unsupervised training interventions showed fewer effects on quality of life or physical function than supervised interventions. $^{15}$ Therefore, partially or remotely supervised, motivational home-based strategies should be tested regarding feasibility and effectiveness. A previous study showed that young patients with cancer have problems to reliably self-reflect levels of physical activity. ${ }^{16}$ While a relevant number of patients overestimated their daily minutes of walking, underestimation occurred same as often. Lacking self-reflection of physical activity levels seems to be an important issue for 
children during cancer treatment. This aspect needs to be taken under consideration when applying exercise programmes. All interventions should be low risk with regard to overload and injuries and individually adapted to the child's physical condition.

The aim of the study was to evaluate the usability of activity trackers and centralised monitoring to conduct a home-based exercise intervention during acute cancer treatment. Because adherence to home-based exercise programmes is often one of the most important aspects to achieve effects on patient-related outcomes, our primary aim was to evaluate achievement of individual goals for daily steps in the intervention group (IG) over time and compared between the IG and the control group (CG). Secondary endpoints included achievement of goals for active minutes, effects of the intervention on motor performance and health-related quality of life (hrQoL) and general perceptions regarding the programme.

\section{MATERIALS AND METHODS \\ Participant recruitment and study design}

Children and adolescents were considered eligible under the following conditions: age between 8 and 17 years, paediatric cancer diagnosis, regular appointments at the hospital, access to an internet-accessible computer or smartphone, ability to communicate in German or English and ability to ambulate. Children for the IG had to fulfil additional criteria: diagnosis after 1 January 2014 and regular inpatient stays. Exclusion criteria included: medical contraindication to walk and to perform individually adapted exercises (eg, bed-rest or reduced load on bones due to tumour or metastasis) identified by the oncologist, more than 8 weeks between hospital appointments and mental disability. The study protocol adheres to the ethics guidelines of the Declaration of Helsinki and was approved by the local Ethics Committee of the General Medical Council Westfalen-Lippe and the Westfälische Wilhelms-Universität Münster (2014-219 f-S). Participants for the IG needed to be in their acute treatment phase and were recruited gradually with respect to limited availability of study instruments and capacities to ensure a high quality of centralised monitoring and supervision. Children for the CG were recruited immediately at study start out of children who had already completed their intensive inpatient treatment phase and at the end of the study out of children who could not have been included in the IG due to recruitment completion. The study contents were explained both orally and in writing and all participants and their parents gave their written informed consent. The study design was a prospective, quasiexperimental study. Children in the IG received a $6-8$ week intervention during treatment (T1) and a 2-week intervention within 3 months after cessation of acute cancer treatment (T2) whereas children in the CG only received the intervention after acute cancer treatment (T2).

\section{Assessments}

Every participant of the IG and CG underwent a baseline assessment before the interventions at $\mathrm{T} 2$ that consisted of measuring number of steps, active minutes per day, motor performance, hrQoL and physical activity levels prior to and during treatment. The IG was additionally tested prior their intervention during cancer treatment (T1). Guided interviews were conducted with the IG during the in-hospital stays between the home stays and with all participants at the end of the study after the 2-week intervention.

\section{Daily steps and active minutes}

Baseline measures on physical activity as well as feedback during the intervention at $\mathrm{T} 1$ and $\mathrm{T} 2$ were performed using the Fitbit monitor (Fitbit, San Francisco, CA). Participants chose between the Fitbit One (worn in the pocket, $4.8 \mathrm{~cm} \times 1.9 \mathrm{~cm} \times 1.0 \mathrm{~cm}$ ) and Fitbit Flex (wristband, $1.5 \mathrm{~cm} \times 1.0 \mathrm{~cm} \times 14-20 \mathrm{~cm})$. These triaxial accelerometers convert acceleration to steps using proprietary algorithms. The Fitbit has been shown to be an accurate tool in a cardiac rehabilitation programme validated against Actigraph accelerometer $(\mathrm{r}=0.95) .{ }^{17}$ A literature search revealed three studies in paediatric cancer populations. One study used the Fitbit to increase physical activity during maintenance steroid pulses ${ }^{18}$ and the other study evaluated a home-based physical activity intervention with the Fitbit in childhood cancer survivors. ${ }^{19}$ The third study used the Fitbit in combination with a Facebook mHealth intervention to increase physical activity in survivors of childhood cancer. ${ }^{20}$ All data are uploaded to a password-protected website. The dashboard summarises step counts, number of active minutes, daily walking distance and calories burned. All participants in this study obtained their log-in email address and password by the study investigators. To define the individual goal for the intervention period, average steps of the 1-week baseline assessment were determined and increased by $+10 \%$. This goal was registered at the account and the Fitbit provided feedback by a vibration signal when achieving it. Participants could also track their status by clicking on the device or looking into the dashboard at their PC or smartphone app.

\section{Motor performance}

Motor performance was determined with the paediatric cancer-specific MOON test (test for motor performance in paediatric oncology). ${ }^{21}$ The test consists of eight subtests: (1) inserting pins (hand-eye coordination under time pressure); (2) static stand (static balance/whole body posture on a wooden bar); (3) reaction test (speed/ reaction on optical stimuli); (4) throwing at a target (coordination with precision of the upper extremity); (5) stand-and-reach (flexibility of hamstring and erector spinae muscles); (6) medicine ball shot (explosive muscle strength of the upper extremity); (7) sit-to-stand (muscular endurance of legs); and (8) handheld dynamometry (isometric maximum hand grip strength). The 
Table 1 Study characteristics

\begin{tabular}{|c|c|c|}
\hline & Intervention group (IG_T2) & Control group (T2) \\
\hline Characteristic & $n=21$ & $n=19$ \\
\hline Age (years) & $14.5 \pm 3.9(15.3 ; 6-23)$ & $15.4 \pm 3.7(17.2 ; 6-20)$ \\
\hline BMI $\left(\mathrm{kg} / \mathrm{m}^{2}\right)$ & $20.3 \pm 4.5(19.4 ; 13-33)$ & $20.2 \pm 3.3(20.5 ; 13-26)$ \\
\hline Male, n (\%) & $13(62)$ & $9(47)$ \\
\hline Months since diagnosis & $9.6 \pm 2.9(9.1 ; 6-17)$ & $8.4 \pm 3.7(6.1 ; 3-16)$ \\
\hline Days since last inpatient ctx & $67.0 \pm 32.3(62.0 ; 6-144)$ & $47.6 \pm 30.8(47.0 ; 1-118)$ \\
\hline \multicolumn{3}{|l|}{ Cancer type } \\
\hline ALL & $9(43 \%)$ & $4(21 \%)$ \\
\hline AML & $1(5 \%)$ & $0(0 \%)$ \\
\hline Lymphoma & $6(29 \%)$ & $5(26 \%)$ \\
\hline EWS & $3(14 \%)$ & $4(21 \%)$ \\
\hline OSS & $1(5 \%)$ & $2(11 \%)$ \\
\hline \multicolumn{3}{|l|}{ Tumour location } \\
\hline Lower extremity & $3(14 \%)$ & $4(21 \%)$ \\
\hline Upper extremity/trunk & $1(5 \%)$ & $1(5 \%)$ \\
\hline Other solid tumour & $1(5 \%)$ & $4(21 \%)$ \\
\hline \multicolumn{3}{|l|}{ Medical therapy } \\
\hline Ctx & $21(100 \%)$ & $19(100 \%)$ \\
\hline Operation & $4(19 \%)$ & $8(42 \%)$ \\
\hline Resection & $3(14 \%)$ & $3(16 \%)$ \\
\hline Prosthesis & $1(5 \%)$ & $4(21 \%)$ \\
\hline Amputation & $0(0 \%)$ & $1(5 \%)$ \\
\hline Radiotherapy & $4(19 \%)$ & $7(37 \%)$ \\
\hline Stem cell transplantation & $2(10 \%)$ & $5(26 \%)$ \\
\hline Autologous & $0(0 \%)$ & $2(11 \%)$ \\
\hline Allogeneic & $2(10 \%)$ & $3(16 \%)$ \\
\hline Partially limited walking ability & $3(14 \%)$ & $5(26 \%)$ \\
\hline
\end{tabular}

Results are given as mean \pm SD (median; range).

ALL, acute lymphoblastic leukaemia; AML, acute myeloic leukaemia; BMI, body mass index; ctx, chemotherapy; EWS, Ewing's sarcoma; IG, intervention group; OSS, osteosarcoma.

whole test takes about $20 \mathrm{~min}$ and contains age/gendermatched reference values (RefV) for each test item separately.

\section{Health-related quality of life}

HrQoL was assessed with the KINDL questionnaire (https://www.kindl.org/english/questionnaires/). This generic instrument consists of 24 items associated with the six dimensions: physical well-being, emotional well-being, self-esteem, family, friends and everyday functioning. The subscales can be analysed separately or be combined to a total score. The present study used the child's version for 7-13 year-olds and the adolescent version for $14-17$ year-olds. ${ }^{22}$

\section{Physical activity behaviour}

We used the physical activity questionnaire ${ }^{23}$ from the German Health Interview and Examination Survey for Children and Adolescents in a modified version that includes questions about physical activity prior to cancer treatment as it has been used previously in paediatric patients during and after cancer treatment. ${ }^{2425}$

\section{Opinions and values}

To gather information about how the patients felt about managing the home-based intervention, all participants of the IG were interviewed after every home stay. Questions were based on the four topics: (1) handling of the Fitbit; (2) reasons for not achieving goals; (3) need for support and any problems or risks occurring; and (4) acceptance of the exercise plan. After T2 and the subsequent intervention for both groups, we interviewed all participants in detail about handling the Fitbit and assessed their general perception about the programme.

\section{Intervention}

Participants of the IG received a $6-8$ week home-based exercise intervention during acute treatment. This 
Table 2 Levels of physical activity at baseline T1 and T2 and mean goals for steps and active minutes

\begin{tabular}{|c|c|c|c|c|c|c|}
\hline & \multicolumn{2}{|l|}{ IG_T1 (n=21) } & \multicolumn{2}{|l|}{ IG_T2 $(n=20)$} & \multicolumn{2}{|l|}{ CG_T2 (n=19) } \\
\hline & Steps & $\begin{array}{l}\text { Active } \\
\text { minutes }\end{array}$ & Steps & Active minutes & Steps & $\begin{array}{l}\text { Active } \\
\text { minutes }\end{array}$ \\
\hline Mean \pm SD & $5328 \pm 2932$ & $14.6 \pm 15.5$ & $6908 \pm 3105$ & $26.4 \pm 25.4$ & $6000 \pm 2974$ & $18.9 \pm 22.5$ \\
\hline Median & 5130 & 8.8 & 6218 & 15.6 & 5865 & 11.8 \\
\hline $25 \% ; 75 \%$ percentiles & $2861 ; 7851$ & $3.5 ; 25.6$ & $4406 ; 9733$ & $9.3 ; 45.1$ & $3274 ; 6875$ & $3.3 ; 21.8$ \\
\hline Range (min; max) & $1363 ; 11295$ & $0 ; 61.2$ & $2840 ; 13185$ & $0 ; 97.1$ & $2381 ; 13038$ & $0 ; 92.1$ \\
\hline Step goals (mean $\pm S D)$ & $5861 \pm 3148$ & $16.1 \pm 17.1$ & $7599 \pm 3416$ & $29.0 \pm 27.9$ & $6600 \pm 3271$ & $20.8 \pm 24.8$ \\
\hline
\end{tabular}

Statistical differences IG_T1 versus IG_T2: $p=0.02$ (steps), $p=0.04$ (active minutes).

Statistical differences IG_T2 versus CG_T2: $p=0.49$ (steps), $p=0.26$ (active minutes).

CG, control group; IG, intervention group.

individual programme was performed by the children autonomously and comprised daily goals for steps and active minutes based on their baseline assessments as well as tailored exercises to improve strength, coordination and endurance. The exercises were chosen based on the results of motor performance testing and their exercise preferences and all exercises were explained and trained once under the supervision of the study coordinator. The exercise plan-illustrated by little pictures-consisted of five to seven exercises organised into two alternating training sessions. As an example, the plan for a 12-year-old boy consisted of the following exercises:

- Stretching of the hamstring muscles for $30 \mathrm{~s}$, daily.

- Balance on one leg for $30 \mathrm{~s}$ per leg, daily.

- Chair rise exercise without using the arms, $3 \times 15$ repetitions, thrice a week.

- Strength exercise for the dorsal musculature with an elastic band, $3 \times 15$ repetitions, thrice a week.

- Explosive push-ups on the wall, $3 \times 10$ repetitions, thrice a week.

Aims for steps and active minutes remained the same during the intervention period while exercises were adapted to give variety. The study participants were encouraged to contact the study coordinator if any questions or problems occurred during the home programme. In addition, the programme was discussed regularly during the periodical inpatient stays. All participants including the CG received the same intervention for 2-3 weeks within the first 3 months after the last inpatient chemotherapy (online supplementary figure 1 ).

\section{Statistical analysis}

Commercial software (GraphPad Prism, V.6.0) was used for all statistical analyses. Patient's characteristics are indicated as mean, SD, median and range. Successful achievement of daily steps and active minutes was determined as a deviation of less than $30 \%$ from the defined goal. Results are given as median of days with less than $30 \%$ deviation. As an example, the individual goal was set with 5.000 daily steps and 10 active minutes per day. This goal was achieved and rated successful, if the participant walked between 3.500 and 6.500 steps and performed between 7 and 13 active minutes. Due to absent normal distribution, all group comparisons were
A

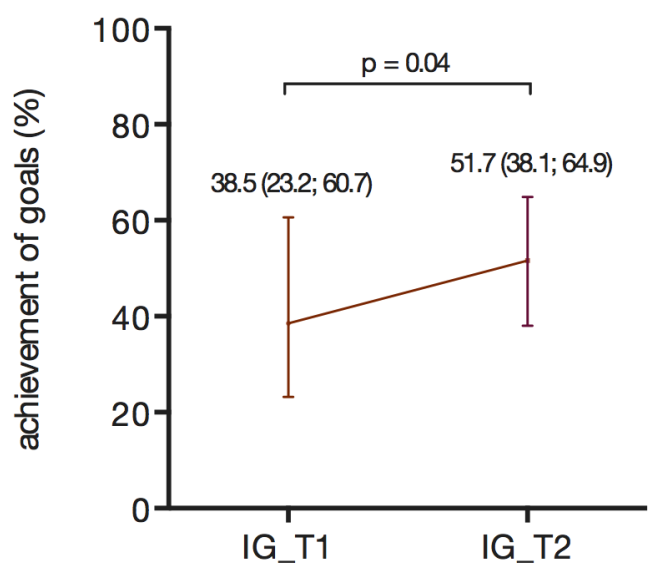

B Daily active minutes

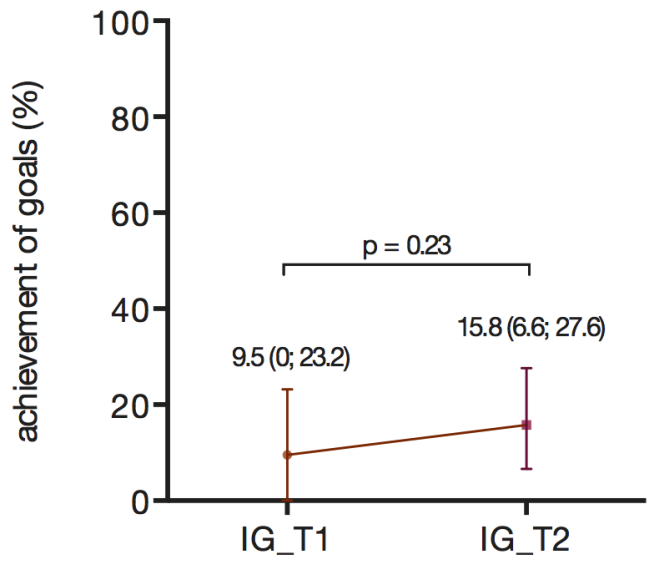

Figure 1 Achievement of individual goals in the intervention group (IG) for steps (A) in a confirmatory analysis and active minutes (B). Results are given as median and range, achievement is defined as less than $30 \%$ deviation from the defined goal. 
Table 3 Results of motor performance (MOON) between IG and CG

\begin{tabular}{|c|c|c|c|c|c|c|c|c|c|}
\hline \multirow[b]{2}{*}{ Test item } & \multirow[b]{2}{*}{$\mathbf{n}$} & \multicolumn{3}{|c|}{ Intervention group (T2) } & \multirow[b]{2}{*}{$\mathbf{n}$} & \multicolumn{3}{|c|}{ Control group (T2) } & \multirow[b]{2}{*}{$P$ values } \\
\hline & & Mean \pm SD & Median & $\begin{array}{l}\text { Patients } \\
\text { below } \\
\text { RefV (\%) }\end{array}$ & & Mean \pm SD & Median & $\begin{array}{l}\text { Patients } \\
\text { below } \\
\text { RefV (\%) }\end{array}$ & \\
\hline Inserting pins & 20 & $-0.7 \pm 8.4$ & -1.7 & 50 & 19 & $-4.7 \pm 15.3$ & -3.4 & 58 & 0.61 \\
\hline Static stance & 19 & $-2.2 \pm 4.6$ & -1.8 & 58 & 19 & $-3.1 \pm 7.5$ & -1.8 & 58 & 0.95 \\
\hline Speed/reaction time & 20 & $-5.2 \pm 8.7$ & -6.3 & 75 & 19 & $-6.6 \pm 9.6$ & -4.3 & 63 & 0.97 \\
\hline Flexibility & 20 & $-10.5 \pm 11.1$ & -9.9 & 80 & 19 & $-7.1 \pm 9.5$ & -8.3 & 79 & 0.51 \\
\hline Explosive strength & 20 & $-26.5 \pm 16.3$ & -25.8 & 90 & 18 & $-32.1 \pm 12.5$ & -31.3 & 100 & 0.27 \\
\hline Muscular endurance legs & 20 & $2.3 \pm 27.6$ & 3.6 & 35 & 19 & $-4.1 \pm 33.6$ & 4.2 & 42 & 0.70 \\
\hline Hand grip strength right & 20 & $-10.3 \pm 29.2$ & -13.6 & 65 & 18 & $-23.9 \pm 25.1$ & -25.5 & 78 & 0.18 \\
\hline Hand grip strength left & 20 & $-12.9 \pm 30.4$ & -16.9 & 70 & 18 & $-23.6 \pm 24.2$ & -21.9 & 83 & 0.35 \\
\hline
\end{tabular}

CG, control group; IG, intervention group; RefV, reference value.

calculated with non-parametric tests (Wilcoxon signedrank test for changes in the IG and Mann-Whitney U test for comparisons between IG and CG). For results of motor performance, the percentage difference of each test item between every single patient and the respective RefV was analysed with the Wilcoxon signed-rank test. The primary endpoint and confirmatory analysis was achievement of individual goals for daily steps, compared (A) in the IG over time and (B) between the IG and CG. In the confirmatory statistical analysis, first the primary endpoint was compared between $\mathrm{T} 1$ and $\mathrm{T} 2$ on local significance level $\alpha=0.05$. If and only if a significant result was attained, in a second step of the primary analysis the IG and CG were compared on local significance level $\alpha=0.05$. This multiple testing procedure keeps the multiple significance level $\alpha=0.05$ and results provide confirmatory statistical evidence. Secondary endpoints included achievement of individual goals for active minutes and effects on motor performance and hrQoL. In the statistical analysis of secondary endpoints, $p$ values are regarded noticeable ('significant') in case $p \leq 0.05$, without adjustment for multiple testing and determination of an overall significance level.

\section{RESULTS}

\section{Study participants}

A consort diagram for recruitment of the IG is shown in the online supplementary figure 2 . The CG only underwent assessments and intervention at the end of acute cancer treatment (T2). Out of 25 contacted patients, $19(73 \%)$ agreed to participate in the CG and no dropouts occurred. Reasons not to participate in the CG were the following: not the time, active themselves, not interested and further surgery needed. Study characteristics are presented in table 1 .

\section{Baseline physical activity}

Physical activity levels measured during baseline assessments are summarised in table 2.

\section{Achievement of individual goals}

Figure 1 illustrates achievement of individual goals for daily steps (A) and daily active minutes (B). Achievement of steps increased from T1 (during treatment) to T2 (shortly after cessation of treatment). Achievement of individual goals for daily active minutes (B) was generally very low and did only increase marginally from $\mathrm{T} 1$ to T2. Group analyses between the IG and CG did not reveal any significant differences regarding achievement of goals (median achievement of step goals IG: $51.7 \%$ vs CG: $44.4 \%, \mathrm{p}=0.48$; median achievement of goals for active minutes IG: $15.8 \%$ vs CG: $15.4 \%, \mathrm{p}=0.76$, graph not shown). Out of all 40 participants, only five children chose the Fitbit one (worn in the pocket) and 35 chose the Fitbit flex (worn at the wrist).

\section{Motor performance}

Impaired domains of motor performance at $\mathrm{T} 1$ in the IG were static balance $(\mathrm{p}=0.026)$, speed/reaction time $(\mathrm{p}=0.013)$, flexibility $(\mathrm{p}<0.001)$, explosive strength $(\mathrm{p}<0.001)$ and maximum hand grip strength of the right hand $(\mathrm{p}=0.048)$. Results of motor performance in the IG did not change significantly between $\mathrm{T} 1$ and $\mathrm{T} 2$, except muscular endurance of the legs (sit-to-stand test), which improved from $\mathrm{T} 1$ to $\mathrm{T} 2(\mathrm{p}<0.0001)$. Comparing the results of motor performance between IG and CG participants did not reveal significant differences (see table 3). However, for all strength tests (explosive strength, muscular endurance of legs and hand grip strength) a higher percentage of CG than IG participants scored values below the reference.

Results are deviations (\%) from age/gendermatched RefV with exception of static stance and flexibility (absolute deviations), $\mathrm{p}$ value based on MannWhitney U test.

\section{Health-related quality of life}

Participants in the CG rated their overall hrQoL significantly lower than IG participants (figure 2A). These differences were particularly present in the 
A

Total score

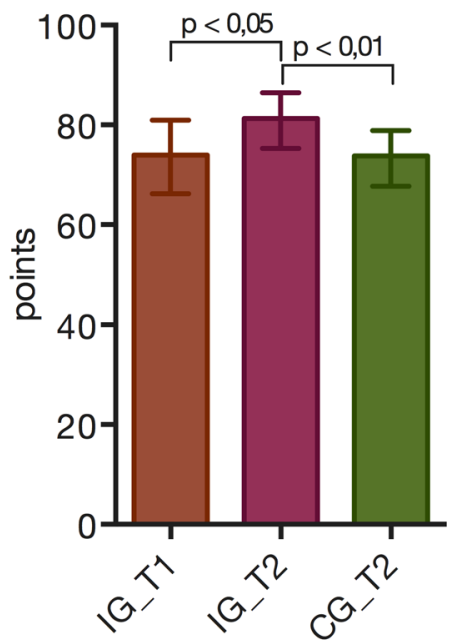

B Physical wellbeing

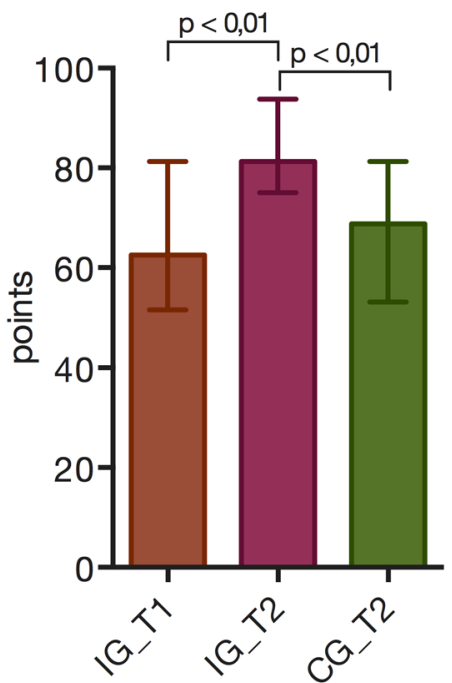

C Self-esteem

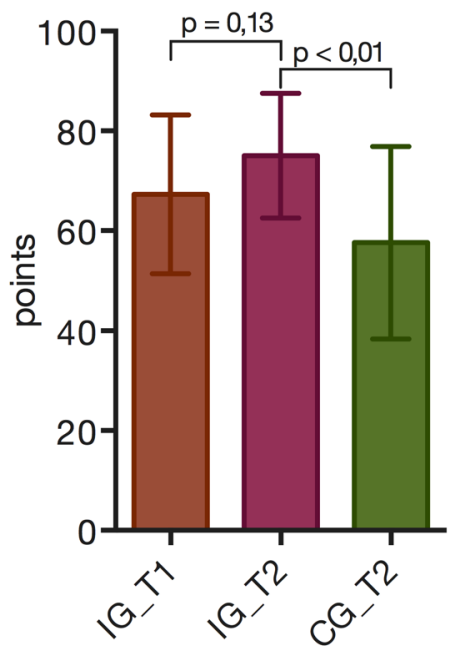

Figure 2 Health-related quality of life in the intervention group (IG) at T1 and T2 and the control group (CG) at T2 for total score $(A)$ and the subdomains physical well-being $(B)$ and self-esteem (C).

subdomains physical well-being (figure 2B) and self-esteem (figure 2C). Results of the remaining subdomains and comparison to respective normative values of healthy children are listed in the online supplementary table 1.

\section{Perceptions and ratings by the participants}

The design of the intervention was perceived as meaningful by $94 \%(34 / 36)$ of participants and all questioned patients recommended this intervention to other children. Eighty per cent $(28 / 35)$ rated the intervention as motivational for increasing physical activity and 23\% (8/35) recognised decreasing motivational effects over time. Patients who did not report increasing motivation suggested that they were sufficiently physically active on their own $(n=2)$, wanted to concentrate on school contents $(n=1)$, did not want to walk more $(n=1)$, felt the need of rewards or competition $(n=1)$, or that their $\operatorname{dog}$ was motivating them more than the tracker $(n=1)$. Most of the patients $(58 \%, 21 / 36)$ felt that they improved their self-reflection of physical activity levels during the study. Eighty-six per cent $(31 / 36)$ of the study participants rated the use of the Fitbit and the provided instructions as easy and simple. Some patients mentioned problems closing the wristband on their own or technical issues.

\section{DISCUSSION}

The study evaluated the feasibility and effects of a homebased intervention using activity trackers and individually tailored exercise plans during cancer treatment. The primary aim was to evaluate patients' achievements of individual goals for daily steps.

\section{Achievement of individual goals}

Achievement of step goals significantly improved from $\mathrm{T} 1$ to T2 in the IG. This phenomenon cannot be explained by an improved physical fitness alone because the goals for steps at T2 were about 1700 steps higher than during treatment and therefore more difficult to achieve. It can be hypothesised that achievement of step goals can be trained over time and by a constant use of feedback. Alternatively, the end of acute cancer treatment positively influenced achievement of goals due to a more constant daily routine, overall generally improved motivation to adhere to the programme and less restrictions compared with acute cancer treatment. This explanation would be supported by the fact that no significant differences were noticed between both groups. There are no comparable interventions available in the current literature that evaluated achievement of step goals. However, two studies tested the applicability of the Fitbit in survivors of paediatric cancer ${ }^{1920}$ and another study used the Fitbit to improve the level of physical activity during a corticosteroid pulse in maintenance therapy. ${ }^{18}$ The most recent study using the Fitbit did not reveal clear differences regarding moderate to vigorous physical activity and sedentary time in the IG wearing the tracker combined with a Facebook mHealth intervention and the $\mathrm{CG}^{20}{ }^{20}$

No increase in achievement of individual goals for active minutes was seen and overall achievement of active minutes was low $(9.5 \%-15.8 \%$, figure 1$)$. While steps are more likely to be integrated into everyday life, active minutes are mostly accumulated during free play or organised sports and require the capability to at least walk fast. This physical prerequisite seems to fluctuate on a daily basis during therapy, which is why baseline testing for active minutes might not even be representative for the next few days. Technically, some activities with at least moderate intensity might have remained undetected because the Fitbit tracker only summarises moderate to 
vigorous activity that lasts at least $60 \mathrm{~s}$. Children during cancer treatment show considerably reduced activity patterns, ${ }^{25} 26$ and activity bouts of less than $60 \mathrm{~s}$ might occur more often than in healthy children.

\section{Perceptions, motor performance and hrQoL}

In general, the children's experience was mainly positive and the intervention was well accepted. This is in accordance with other studies evaluating exercise needs and preferences in children and adolescents with cancer. ${ }^{27} 28$ The IG rated their hrQoL considerably higher at the end of treatment in comparison to the CG. Current evidence about effects of exercise on hrQoL in children and adolescents with cancer is inconclusive: some studies revealed beneficial effects of exercise interventions on hrQoL ${ }^{2029} 30$ while others did not see differences between the exercise and a CG. ${ }^{31}{ }^{32}$ However, findings from systematic reviews in adult oncology mostly revealed positive effects of exercise on improvements of hrQoL. ${ }^{33} \mathrm{In}$ our study, self-esteem was significantly higher, which may be due to the design of the programme that is based on achievement of goals and self-administered exercise.

The individual-adapted home-based exercises, however, did not show a pronounced beneficial effect on motor performance, which might be due to compliance problems in the home-based programme. Some improvements were seen in strength parameters; however, the differences in the CG were not significant. Findings from the literature are inconsistent on this topic. Since some studies including exercise programmes, like the study from Thorsteinsson et al, could only show improvements in physical fitness and physical function 1 year after cessation of treatment but not during intensive treatment, ${ }^{7}$ other studies saw beneficial effects on strength during treatment already. ${ }^{32}$ Possibly, the individual-adapted exercise programme in our study was not performed at home as planned and might need closer supervision. Future approaches might evaluate innovative programmes like web-based exercise programmes to partially supervise the exercise sessions. Another promising solution might be a close cooperation with local exercise professionals like physical education teachers or coaches at sport clubs to provide continuous support during home stays.

\section{Limitations}

Some limitations need to be acknowledged. Allocation to the intervention and the CG was not randomised but dependent on the treatment phase when starting the study. Therefore, no baseline assessment in the CG could be performed during treatment. Nonetheless, this recruitment design enables a representative cohort of children in both groups because participants were not selected by the study investigators intentionally for one group, but based on their treatment phase. Due to the missing baseline test in the CG during treatment, differences between IG and CG at T2 need to be interpreted with caution. Despite some differences in patients' characteristics between the CG and IG, no significant differences were seen regarding physical activity levels. Since patients with cancer with physical impairments show considerably lower levels of physical activity, ${ }^{34}$ our groups seem to be largely comparable. Due to time conflicts or loss of contact, only 36 out of the 40 participating patients could be interviewed at the end of the study. It cannot be precluded that season of year influenced the results. Rain and cold weather may keep children from playing and move outside. However, their individual goals were determined by measuring their activity levels only days prior to the intervention. Primary endpoint was the achievement of these goals and not the amount of steps per se.

\section{CONCLUSION AND PERSPECTIVES}

In conclusion, this home-based intervention was a feasible attempt to close the gap between in-hospital supervised exercise promotion and supply for survivors of childhood cancer. Results of the study indicate that this presented exercise intervention for home stays under central supervision is feasible during and after cancer treatment. Although our intervention did not aim at increasing physical activity levels, children in the IG presented higher activity levels than children in the CG. More important, the study showed how children are able to increase their motivation to achieve individual goals for physical activity. Besides positive effects on hrQoL, further strategies are needed to strengthen positive effects on motor performance. Future investigations should aim at analysing the clinical and motivational influences on physical activity and achievement of goals to better understand the underlying mechanisms and develop appropriate strategies.

\section{Author affiliations}

${ }^{1}$ Department of Pediatric Hematology/Oncology, Center for Child and Adolescent Medicine, University Hospital Essen, Essen, Germany

${ }^{2}$ Department for Pediatric Hematology and Oncology, University Hospital Münster, Münster, Germany

${ }^{3}$ Department of Sport and Health Science, Institute of Preventive Pediatrics, Technical University of Munich, Munich, Germany

${ }^{4}$ Division of Pediatric Hematology/Oncology, Department of Pediatrics,

Comprehensive Cancer Center Munich, Kinderklinik München Schwabing, Klinikum Rechts der Isar, Technical University of Munich, Munich, Germany

${ }^{5}$ Institute of Biostatistics and Clinical Research, University Hospital Münster,

Münster, Germany

${ }^{6}$ Institute of Experimental Musculoskeletal Medicine, University Hospital Münster, Münster, Germany

Acknowledgements We thank the Margarete-Clemens Foundation for financing this study.

Contributors Planning of the study: MG, SVK, JB, DR. Conduct of the study: MG, SVK. Analysis: MG, SVK, JG. Interpretation: MG, SVK, JB, DR. Writing the manuscript: MG, SVK, JB, DR, JG. Final approval: MG, SVK, JB, DR, JG. MG and SVK contributed equally to this paper.

Funding This study was funded by Margarete-Clemens Foundation (T400/25015/2014).

Competing interests None declared.

Patient consent Parental/guardian consent obtained.

Ethics approval Local ethics committee, Ethik-Kommission der Ärztekammer Westfalen-Lippe and the Westfälische Wilhelms-Universität.

Provenance and peer review Not commissioned; externally peer reviewed. 
Open Access This is an Open Access article distributed in accordance with the Creative Commons Attribution Non Commercial (CC BY-NC 4.0) license, which permits others to distribute, remix, adapt, build upon this work non-commercially, and license their derivative works on different terms, provided the original work is properly cited and the use is non-commercial. See: http://creativecommons.org/ licenses/by-nc/4.0/

C) Article author(s) (or their employer(s) unless otherwise stated in the text of the article) 2018. All rights reserved. No commercial use is permitted unless otherwise expressly granted.

\section{REFERENCES}

1. Christiansen JR, Kanellopoulos A, Lund MB, et al. Impaired exercise capacity and left ventricular function in long-term adult survivors of childhood acute lymphoblastic leukemia. Pediatr Blood Cancer 2015;62:1437-43.

2. Armstrong GT, Kawashima T, Leisenring W, et al. Aging and risk of severe, disabling, life-threatening, and fatal events in the childhood cancer survivor study. J Clin Oncol 2014;32:1218-27.

3. Zeltzer LK, Recklitis C, Buchbinder D, et al. Psychological status in childhood cancer survivors: a report from the Childhood Cancer Survivor Study. J Clin Oncol 2009;27:2396-404.

4. Deisenroth A, Söntgerath R, Schuster AJ, et al. Muscle strength and quality of life in patients with childhood cancer at early phase of primary treatment. Pediatr Hematol Oncol 2016;33:393-407.

5. Götte M, Kesting SV, Winter CC, et al. Motor performance in children and adolescents with cancer at the end of acute treatment phase. Eur J Pediatr 2015;174:791-9.

6. Wilson CL, Gawade PL, Ness KK. Impairments that influence physical function among survivors of childhood cancer. Children 2015;2:1-36.

7. Thorsteinsson T, Larsen HB, Schmiegelow K, et al. Cardiorespiratory fitness and physical function in children with cancer from diagnosis throughout treatment. BMJ Open Sport Exerc Med 2017;3:e000179.

8. Cramp F, Byron-Daniel J. Exercise for the management of cancer-related fatigue in adults. Cochrane Database Syst Rev 2012;11:CD006145.

9. Irwin ML, Cartmel B, Gross CP, et al. Randomized exercise trial of aromatase inhibitor-induced arthralgia in breast cancer survivors. J Clin Oncol 2015;33:1104-11.

10. Braam KI, van der Torre P, Takken T, et al. Physical exercise training interventions for children and young adults during and after treatment for childhood cancer. Cochrane Database Syst Rev 2016;3:CD008796

11. Götte M, Taraks S, Boos J. Sports in pediatric oncology: the role(s) of physical activity for children with cancer. J Pediatr Hematol Oncol 2014;36:85-90.

12. Baumann FT, Bloch W, Beulertz J. Clinical exercise interventions in pediatric oncology: a systematic review. Pediatr Res 2013;74:366-74.

13. Rustler V, Hagerty M, Daeggelmann J, et al. Exercise interventions for patients with pediatric cancer during inpatient acute care: A systematic review of literature. Pediatr Blood Cancer 2017;64:e26567

14. Grimshaw SL, Taylor NF, Shields N. The feasibility of physical activity interventions during the intense treatment phase for children and adolescents with cancer: a systematic review. Pediatr Blood Cancer 2016;1463:1586-93.

15. Buffart LM, Kalter J, Sweegers MG, et al. Effects and moderators of exercise on quality of life and physical function in patients with cancer: An individual patient data meta-analysis of 34 RCTs. Cancer Treat Rev 2017;52:91-104.
16. Götte M, Seidel CC, Kesting SV, et al. Objectively measured versus self-reported physical activity in children and adolescents with cancer. PLoS One 2017;12:e0172216.

17. Alharbi $M$, Bauman $A$, Neubeck $L$, et al. Validation of fitbit-flex as a measure of free-living physical activity in a community-based phase III cardiac rehabilitation population. Eur J Prev Cardiol 2016;23:1476-85.

18. Hooke MC, Gilchrist L, Tanner L, et al. Use of a fitness tracker to promote physical activity in children with acute lymphoblastic leukemia. Pediatr Blood Cancer 2016;63:684-9.

19. Le A, Mitchell HR, Zheng DJ, et al. A home-based physical activity intervention using activity trackers in survivors of childhood cancer: a pilot study. Pediatr Blood Cancer 2017;64:387-94.

20. Mendoza JA, Baker KS, Moreno MA, et al. A fitbit and facebook mhealth intervention for promoting physical activity among adolescent and young adult childhood cancer survivors: a pilot study. Pediatr Blood Cancer 2017;64:e26660.

21. Götte M, Kesting S, Albrecht C, et al. MOON-test - determination of motor performance in the pediatric oncology. Klin Padiatr 2013;225:133-7.

22. Ravens-Sieberer U, Gosch A, Abel T, et al. . Quality of life in children and adolescents: a European public health perspective. Soz Praventivmed 2001;46:294-302.

23. Bös K, Worth A, Opper E, et al. Motorik-modul: eine studie zur motorischen leistungsfähigkeit und körperlich-sportlichen aktivität von kindern und jugendlichen in Deutschland. Baden-Baden: Nomos Verlag, 2009.

24. Kesting SV, Götte M, Seidel CC, et al. One in four questioned children faces problems regarding reintegration into physical education at school after treatment for pediatric cancer. Pediatr Blood Cancer 2016;63:737-9.

25. Götte M, Kesting S, Winter C, et al. Comparison of self-reported physical activity in children and adolescents before and during cancer treatment. Pediatr Blood Cancer 2014;61:1023-8.

26. Winter $C$, Müller $C$, Brandes $M$, et al. Level of activity in children undergoing cancer treatment. Pediatr Blood Cancer 2009;53:438-43.

27. Götte M, Kesting S, Winter C, et al. Experience of barriers and motivations for physical activities and exercise during treatment of pediatric patients with cancer. Pediatr Blood Cancer 2014;61:1632-7.

28. Sawyer SM, McNeil R, McCarthy M, et al. Unmet need for healthcare services in adolescents and young adults with cancer and their parent carers. Support Care Cancer 2017;25:2229-39.

29. Speyer E, Herbinet A, Vuillemin A, et al. Effect of adapted physical activity sessions in the hospital on health-related quality of life for children with cancer: a cross-over randomized trial. Pediatr Blood Cancer 2010;55:1160-6.

30. Rosenhagen A, Bernhörster M, Vogt L, et al. Implementation of structured physical activity in the pediatric stem cell transplantation. Klin Padiatr 2011;223:147-51.

31. van Dijk-Lokkart EM, Braam KI, van Dulmen-den Broeder E, et al. Effects of a combined physical and psychosocial intervention program for childhood cancer patients on quality of life and psychosocial functioning: results of the QLIM randomized clinical trial. Psychooncology 2016;25:815-22.

32. Fiuza-Luces C, Padilla JR, Soares-Miranda L, et al. Exercise intervention in pediatric patients with solid tumors: the physical activity in pediatric cancer trial. Med Sci Sports Exerc 2017;49:223-30.

33. Scott K, Posmontier B. Exercise interventions to reduce cancerrelated fatigue and improve health-related quality of life in cancer patients. Holist Nurs Pract 2017;31:66-79.

34. Rueegg CS, Gianinazzi ME, Michel G, et al. . Do childhood cancer survivors with physical performance limitations reach healthy activity levels? Pediatr Blood Cancer 2013;60:1714-20. 